\title{
Intracranial Vertebral Artery Dissection Presenting with Isolated Trigeminal Neuralgia-like Facial Pain
}

\author{
Hanna Choi, Do-Hyung Kim, Sung-Yeon Sohn, Jae Guk Kim and Soo Joo Lee
}

Department of Neurology, Eulji University Hospital, Eulji University School of Medicine, Daejeon, Republic of Korea

Corresponding author: Soo Joo Lee, Department of Neurology, Eulji University Hospital, Eulji University School of Medicine, Daejeon, Republic of Korea, Tel: +82-42-611-3430; E-mail: sjoolee@eulji.ac.kr

Received: Nov 30, 2016; Accepted: Dec 12, 2016; Published: Dec 16, 2016

Citation: Choi H, Kim DH, Sohn SY, et al. Intracranial Vertebral Artery Dissection Presenting with Isolated Trigeminal Neuralgia-like Facial Pain. J Neurol Neurosci. 2016, 7:6.

\begin{abstract}
Headache often accompanies intracranial vertebral artery dissection (IVAD), and infrequently, it is the only symptom. Furthermore, IVAD presenting with isolated facial pain is rarer. Ischemia of the spinothalamic tract caused by IVAD is considered as the cause of facial pain. We present a case of intracranial vertebral artery dissection with trigeminal neuralgia-like facial pain which was successfully treated with antiplatelet agents and Carbamazepine.
\end{abstract}

Keywords: Vertebral artery dissection; Trigeminal neuralgia; Facial pain; Spinothalamic tract

\section{Introduction}

Intracranial vertebral artery dissection (IVAD) [1] is most frequently accompanied by headache, which is infrequently the only symptom [2]. However, IVAD rarely presents with isolated facial pain; trigeminal neuralgia-like facial pain is even rarer, and only a single case has been reported in 2015 [3].

The author presents a case of IVAD in a patient with isolated trigeminal neuralgia-like facial pain without neurological deficits. The patient was successfully treated with antithrombotic therapy without any complications.

\section{Case Report}

A 30-year-old woman was admitted to the emergency room as she experienced right-sided facial pain 4 days prior to admission. The patient reported a sharp pain in the back of the head while abruptly turning it, a week earlier. The pain was momentary and the patient did not remember the side on which the pain was dominant. Four days before the emergency room visit, the patient experienced a sharp piercing pain predominantly on the right side, specifically in the right forehead and right cheek. The part of the body the patient described was in the first and second branches of the right trigeminal nerve. In the absence of any stimulus, she felt infrequent pain that was rated 6 on the numeral rating scale (NRS); she experienced pain rated 8 on the NRS when the part was touched or while chewing food. Other symptoms, such as nausea, vomiting, or dizziness, were not apparent. The vital signs were assessed to be normal, and neurological examination did not reveal deficits such as diplopia or ataxia.

The initial computed tomography angiography (CTA) taken in the emergency room showed focal moderate stenosis and post-stenotic ectasia in the $4^{\text {th }}$ branch of the right vertebral artery (Figure 1). The patient underwent magnetic resonance angiography (MRA) as well as a blood test upon admission, to test for vasculitis. The blood test revealed no abnormalities suggestive of vasculitis. MRA did not show lesions of the right trigeminal nerve itself or lesions compressing the right trigeminal nerve. Stenosis and post-stenotic ectasia in the $4^{\text {th }}$ branch of the right vertebral artery were still observed, and double lumen was suspected in the area (Figure 2). High signal intensity suggestive of acute cerebral infarction was not observed on diffusion-weighted images.

The author suspected right vertebral artery dissection, and prescribed aspirin, Clopidogrel, and fluid therapy, as well as Carbamazepine for pain control. The facial pain decreased to an NRS rating of 1 in 1 day. The patient was discharged, maintained on aspirin, Clopidogrel, and Carbamazepine therapy, and did not show any neurological deterioration during the course of outpatient treatment and follow-up. Three months after the occurrence of the first symptoms, CTA revealed mild stenosis on the right vertebral artery, based on which the stenosis was determined to have improved as compared to that observed in the previous CTA.

\section{Discussion and Conclusion}

The patient had sharp, infrequent, and momentary pain in the area corresponding to the first and second branches of the right trigeminal nerve. Trigeminal neuralgia was suspected because the pain was induced upon the application of a harmless stimulus. At the same time, double lumen was suspected because both CTA and MRA showed local moderate stenosis and post-stenotic ectasia in the $4^{\text {th }}$ branch of the right vertebral artery. Therefore, the patient was diagnosed with right vertebral artery dissection. 


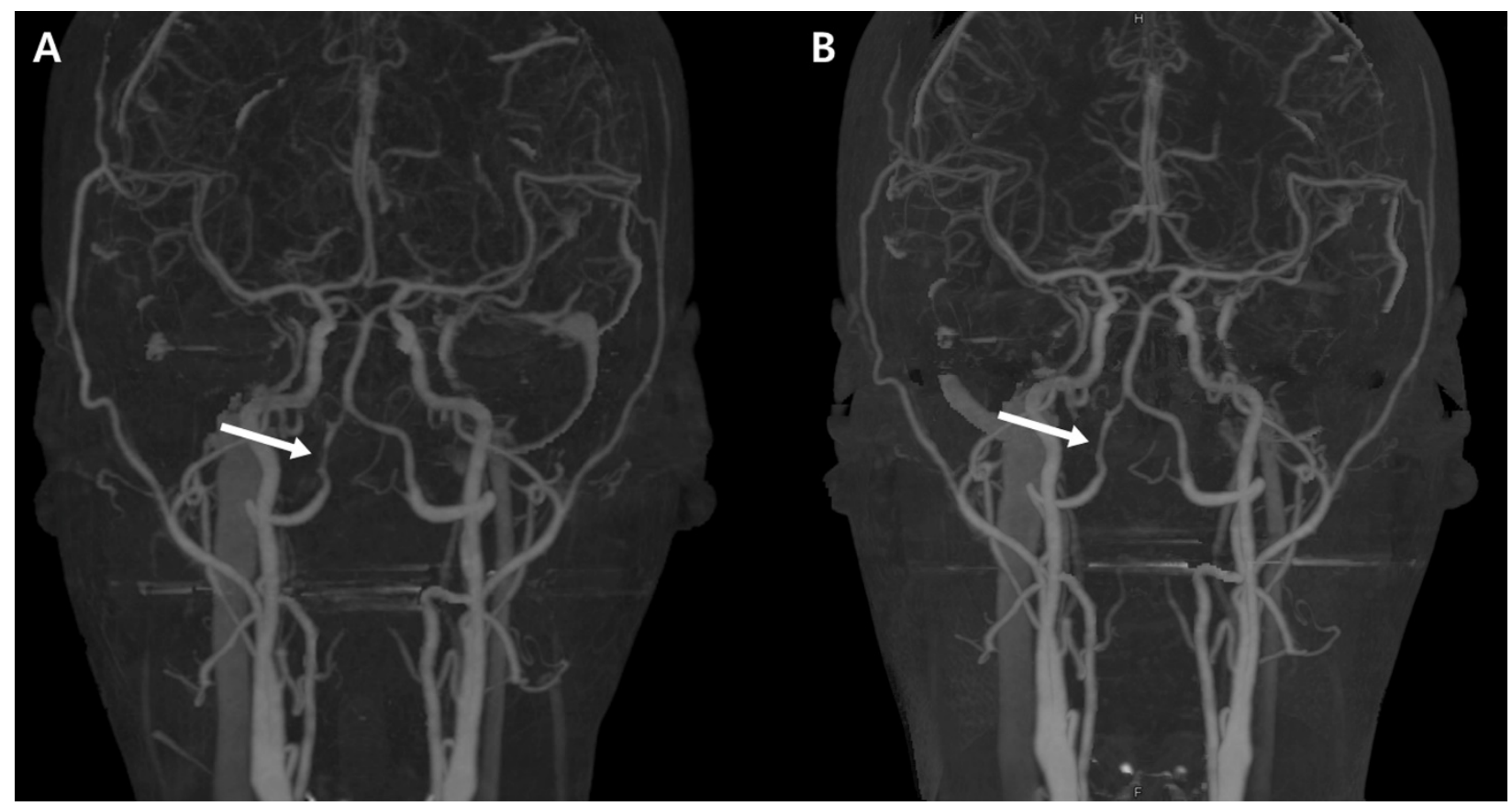

Figure 1 (A) Initial computed tomography angiography (CTA) showed segmental moderate stenosis in the $4^{\text {th }}$ segment of the right vertebral artery (arrow), with post-stenotic ectasia. (B) Follow-up CTA performed approximately 3 months after the initial scan. The degree of stenosis had improved (arrow) compared to that observed on the previous image.

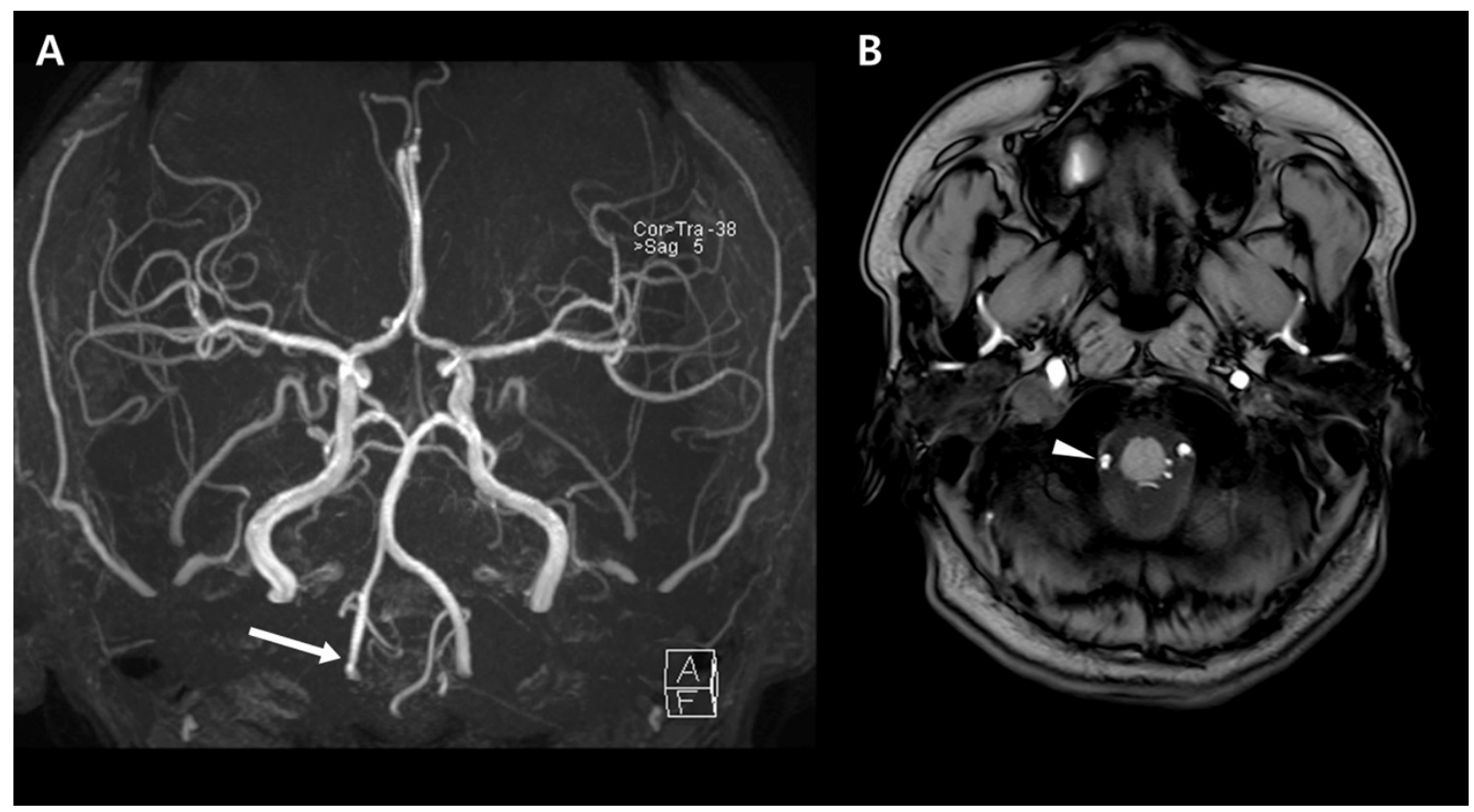

Figure 2 (A) Magnetic resonance angiography (MRA) showed segmental moderate stenosis in the $4^{\text {th }}$ segment of the right vertebral artery (arrow), with post-stenotic ectasia. (B) The double lumen (arrow head) was suspected on an axial time-offlight (TOF) image at the stenotic level.

According to Hosoya et al. the initial symptoms of IVAD most frequently involve severe headache and dizziness (39\%), followed by nausea and vomiting (32\%), dysarthria, tinnitus, and diplopia (14\% each) [1]. Cases of IVAD presenting with facial pain are rare, with only 10 cases reported so far [3-11]. Eight of these cases were accompanied by symptoms or signs of medullary ischemia (e.g., dizziness, [4-7,10,11] ataxia, $[6-8,10,11]$ and sensory abnormalities $[6,8,10,11])$; medullary 
infarction was confirmed on MRA, which helped the diagnosis of vertebral artery dissection.

Only 2 cases of isolated facial pain without the symptoms or signs of medullary ischemia have been reported $[3,9]$. While one involved facial pain that mimicked cluster headaches, [9] the other involved facial pain presenting as trigeminal neuralgia, same as that observed in the present case [3]. In the latter case, despite the confirmation of vertebral artery dissection through MRA, only simple analgesics were prescribed and the facial pain gradually improved over the course of 4 weeks. Although vertebral artery stenosis was shown to have been aggravated on the MRA after the pain disappeared, analgesics were stopped and the course was observed without the prescription of antithrombotic medication. After 3 weeks passed, the patient once experienced dizziness for the duration of several minutes. The MRA taken at that time confirmed that the vertebral artery stenosis was further aggravated, and aspirin was prescribed. The patient did not feel abnormal symptoms thereafter, but an MRA performed 6 months later showed the complete occlusion of the vertebral artery.

Prior to this case, another involving trigeminal neuralgia-like facial pain in a patient with lateral medullary infarction and no vertebral artery dissection had been reported, [12] in which MRA confirmed that the cerebral infarction had intruded into the spinothalamic tract. As a result, the authors assumed that ischemia of the spinothalamic tract induced the pain. Based on other case reports of facial pain without the features of trigeminal neuralgia in patients with lateral medullary infarction and without vertebral artery dissection, $[13,14]$ ischemic lesions of the spinothalamic tract are presumably related to facial pain.

Accordingly, if the MRA of a patient with abrupt facial pain shows signs of IVAD even without lateral medullary infarction, ischemia of the spinothalamic tract should be suspected. In such cases, unless prescribing antithrombotic medications is contraindicated, antithrombotic therapy is recommended. In the present case, unlike that reported by Nakamizo et al., antithrombotic treatment began immediately upon the diagnosis of vertebral artery dissection; Carbamazepine, not simple analgesics, was prescribed to control the pain. The NRS status of the patient's facial pain decreased from 8 to 1 in 1 day, and no complications such as dizziness, ataxia, or diplopia occurred during the follow-up period. Such rapid recovery could have been due to Carbamazepine, which is known as the primary medication used to treat trigeminal neuralgia [15]. At the same time, ischemia caused by vertebral artery dissection has also been successfully treated with antithrombotic medication and fluid therapy, and might contribute to the recovery.

Altogether, IVAD can present with facial pain, sometimes with the features of trigeminal neuralgia, without any neurological deficits. Vertebral artery dissection could be the cause of abrupt facial pain accompanying trigeminal neuralgia.
With the confirmation through MRA findings, even if lateral medullary infarction is not observed, vertebral artery dissection should be treated with antithrombotic therapy, both to prevent cerebral infarction caused by the vertebral artery dissection as well as to relieve facial pain.

\section{References}

1. Hosoya $\mathrm{T}$, Adachi $\mathrm{M}$, Yamaguchi $\mathrm{K}$, Haku $\mathrm{T}$, Kayama $\mathrm{T}$, et al. (1999) Clinical and neuroradiological features of intracranial vertebrobasilar artery dissection. Stroke 30: 1083-1090.

2. Maruyama H, Nagoya H, Kato $Y$ (2012) Spontaneous cervicocephalic arterial dissection with headache and neck pain as the only symptom. J Headache Pain 13: 247-253.

3. Nakamizo T, Koide T, Miyazaki H (2015) Progressive Intracranial Vertebral Artery Dissection Presenting with Isolated Trigeminal Neuralgia-Like Facial Pain. Case Rep Neurol Med 2015: 387139.

4. Canepa Raggio C, Dasgupta A (2014) Three cases of spontaneous vertebral artery dissection (SVAD), resulting in two cases of Wallenberg syndrome and one case of Foville syndrome in young, healthy men. BMJ Case Rep 2014.

5. Cremer PD, Halmagyi GM, Goadsby PJ (1995) Secondary cluster headache responsive to sumatriptan. J Neurol Neurosurg Psychiatry 59: 633-634.

6. De La Sayette V, Leproux F, Letellier P (1999) Cervical cord and dorsal medullary infarction presenting with retro-orbital pain. Neurology 53: 632-634.

7. Galende AV, Camacho A, Gomez EC (2004) Lateral medullary infarction secondary to vertebral artery dissection presenting as a trigeminal autonomic cephalalgia. Headache 44: 70-74.

8. Jacob S, Rajabally Y (2007) Short-lasting unilateral neuralgiform headache with cranial autonomic symptoms (SUNA) following vertebral artery dissection. Cephalalgia 27: 283-285.

9. Kim JT, Lee SH, Choi SM (2008) Spontaneous vertebral artery dissection mimicking cluster headache. Cephalalgia 28: 671-673.

10. Lai SL, Chang YY, Liu JS, Chen SS (2005) Cluster-like headache from vertebral artery dissection: angiographic evidence of neurovascular activation. Cephalalgia 25: 629-632.

11. Ordas CM, Cuadrado ML, Simal P (2011) Wallenberg's syndrome and symptomatic trigeminal neuralgia. J Headache Pain 12: 377-380.

12. Warren HG, Kotsenas AL, Czervionke LF (2006) Trigeminal and concurrent glossopharyngeal neuralgia secondary to lateral medullary infarction. Am J Neuroradiol 27: 705-707.

13. Altavilla R, Landi D, Altamura C (2012) Dorsolateral medullary ischemic infarction causing autonomic dysfunction and headache: a case report. J Headache Pain 13: 327-330.

14. Cid CG, Berciano J, Pascual J (2000) Retro-ocular headache with autonomic features resembling "continuous" cluster headache in lateral medullary infarction. J Neurol Neurosurg Psychiatry 69: 134.

15. Cruccu G, Gronseth G, Alksne J (2008) AAN-EFNS guidelines on trigeminal neuralgia management. Eur J Neurol 15: 1013-1028. 